\title{
Diagnostic utility of a p63/ $\alpha$-methyl-CoA- racemase (p504s) cocktail in atypical foci in the prostate
}

\author{
Vincent Molinié ${ }^{1}$, Gaëlle Fromont ${ }^{2}$, Mathilde Sibony ${ }^{3}$, Annick Vieillefond ${ }^{4}$, \\ Viorel Vassiliu ${ }^{5}$, Béatrix Cochand-Priollet ${ }^{6}$, Jean M Hervé ${ }^{7}$, Thierry Lebret $^{7}$ and \\ Anne C Baglin ${ }^{1}$
}

${ }^{1}$ Service de Pathologie, Hôpital Foch, Suresnes, France; ${ }^{2}$ Service de Pathologie, Institut Mutualiste Montsouris, Paris, France; ${ }^{3}$ Service de Pathologie, Hôpital Tenon, Paris, France; ${ }^{4}$ Service de Pathologie, Hôpital Cochin, Paris, France; ${ }^{5}$ Service de Pathologie, Hôpital Necker, Paris, France; ${ }^{6}$ Service de Pathologie, Hôpital Lariboisière, Paris, France and ${ }^{7}$ Service d'Urologie, Hôpital Foch, Suresnes, France

\begin{abstract}
Prostatic needle biopsy is the preferred method for diagnosing early prostate cancer, providing specific information. In cases of histological cancer mimics, a diagnosis of atypical small acinar proliferation suspected of but not diagnosed as malignancy can be made. In such cases, and in small focus carcinomas, pathologists use $34 \beta E 12$, cytokeratin (CK) $5 / 6$ or p63 immunostaining to label basal cells, and $\alpha$-methylacyl-CoA racemase (AMACR/p504s) immunostaining as a positive prostate cancer marker on two distinct slides. However, in cases of small foci, ambiguous lesions might disappear. The purpose of our study was to improve the sensitivity of a cocktail of two antibodies (p63/p504s) with a sample incubation on 260 prostatic specimens, in order to help make a decision in conjunction with standard histology and CK 5/6 immunostaining. We tested 101 small focus prostatic cancers, 104 atypical small acinar proliferation, 19 high-grade prostatic intraepithelial neoplasia, two atypical adenomatous hyperplasia and 34 benign mimics of cancer. After p63/p504s immunostaining, the final diagnoses retained were as follows: 154 prostatic cancers, 14 atypical small acinar proliferation, 30 high-grade prostatic intraepithelial neoplasia, three atypical adenomatous hyperplasia and 62 benign mimics of cancer. To differentiate malignant from benign lesions, we used the criteria of greater sensitivity to p504s/p63 (95\%) than to CK 5/6 (57\%) or p63 (86\%), and higher specificity for p504s/p63 (95\%) than for CK 5/6 (88\%) or p63 (81\%). With the p504s/p63 cocktail, $89 \%$ of the ambiguous lesions were classified vs $53 \%$ for CK $5 / 6$. Combined use of the two antibodies, one (p504s) as a positive marker and the other (p63) as a negative marker, with a simple immunostaining procedure, may improve diagnostic performance, sensitivity and specificity, leading to a reduction in the risk of false negatives; this technique in cases of atypical small acinar proliferation should reduce the percentage of residual ambiguous lesions and the need for additional biopsies. Modern Pathology (2004) 17, 1180-1190, advance online publication, 18 June 2004; doi:10.1038/modpathol.3800197
\end{abstract}

Keywords: prostate cancer; $\alpha$-methyl-CoA-racemase; prostate biopsy; ASAP; immunohistochemistry

Prostate cancer is one of the most common malignant diseases for which health-care intervention is sought worldwide, and in many developed countries it is the most common noncutaneous malignant disease. ${ }^{1}$ Prostatic needle biopsy is the preferred method for diagnosing early prostate cancer; it has low morbidity and provides specific information on the grade and extent of the tumor. However, in some cases an ambiguous diagnosis is made. The diffi-

Correspondence: Dr V Molinié, MD, Département de Pathologie, Hôpital Foch, 40 Rue Worth, 92151 Suresnes Cedex, France.

E-mail: v.molinie@hopital-foch.org

Received 23 December 2003; revised 18 February 2004; accepted 19 February 2004; published online 18 June 2004 culty with needle biopsy stems not only from the small amount of tissue available for histological examination, but also from the fact that biopsies often identify only a few malignant glands (small focus carcinomas, minute carcinomas) or several histological benign mimics of cancer (Table 1).

Since one feature distinguishing cancer mimics from prostate cancers is that benign glands contain basal cells, which are absent in cancerous glands, pathologists often use immunohistochemical markers to label basal cells when faced with an ambiguous lesion. ${ }^{2}$ The most commonly used marker is the high-molecular-weight cytokeratin (CK) 903 (34ßE12) and, more recently, markers such as CK 5/6 and p63 have been proposed. ${ }^{3-5}$ More 
Table 1 Benign mimics of prostate adenocarcinoma

Adenosis (atypical adenomatous hyperplasia)

Atrophy

Basal cell hyperplasia

Cowper's glands

Mesonephric hyperplasia

Nephrogenic adenoma

Radiation atypia

Seminal vesicles

Verumontanum hyperplasia

Clear-cell cribriform hyperplasia

Xanthoma

recently, $\alpha$-methylacyl-CoA racemase (AMACR) has been reported to be a new marker of prostate cancer. ${ }^{6}$ Although its role in prostatic carcinogenesis is unclear, several recent studies have shown that AMACR expression is upregulated in prostatic cancer, and in immunohistochemistry, 97-100\% of prostate cancers are positive for AMACR. As a result, AMACR has been proposed as a positive prostate cancer marker in conjunction with negative basal cell markers (high-molecular-weight CK or p63) in the workup of difficult prostate needle biopsies. Most of the previous studies were performed with the two antibodies on two distinct slides, and in some cases, especially in small foci, the ambiguous lesion could disappeared on further slides required for immunohistochemical studies. To our knowledge, only $\mathrm{Luo}^{7}$ performed a doublestep incubation with p63 and AMACR, but their overnight incubation period is not suitable for routine diagnostic procedures. Therefore, the purpose of the present study to test a cocktail of two antibodies, p63 and AMACR (p504s), on 260 prostate specimens, was to modified the protocol proposed by Luo et $a l^{8}$ in order to reduce the time it takes, and to demonstrate the technical feasibility of such methods for routine procedures using singlestep incubation on an automatic immunostaining processor.

\section{Materials and methods}

\section{Case Selection}

Among 1640 consecutive prostate specimens from surgical pathological files of six French Parisian hospitals from May to August 2003, we selected 260 cases of small prostate cancer foci or ambiguous lesions, observed in 217 needle biopsies, 12 transurethral prostatic resections, eight adenomectomies, and 23 radical prostatectomies (RP) (Table 2).

\section{Immunohistochemistry for AMACR, CK 5/6 and p63}

Immunohistochemistry for AMACR was performed using a monoclonal anti-AMACR antibody (p504S, $0.5 \mu \mathrm{g} / \mathrm{ml}$, Zeta Corporation, Sierra Madre, CA,
Table 2 Type of specimens

Transurethral resection

Adenomectomy

Radical prostatectomy

USA), a monoclonal anti-p63 antibody (1:80 dilution, Dako, Paris, France) and a primary antibody against high-molecular-weight CK (CK 5/6, 1:80 dilution, Chemicon Int., Tenaculo, CA, USA). Briefly, tissue sections were antigen-retrieved in $0.1 \mathrm{M}$ citrate buffer, $\mathrm{pH} 6.0$, in a hot steamer for $30 \mathrm{~min}$. The immunostaining process was performed on a Ventana Nexes ${ }^{\circledR}$ processor at $37^{\circ} \mathrm{C}$. Subsequently, slides were incubated with the primary CK $5 / 6$ or cocktail antibody (p504S/p63), biotinylated secondary anti-mouse anti-rabbit mixed antibody with the same avidin-peroxidase complex (Ventana), and chromogenic substrate diaminobenzidine; slides were then counterstained with hematoxylin.

\section{Evaluation for Immunohistochemistry}

In all cases, hematoxylin and eosin saffron and immunohistochemistry for CK 5/6 and p63/p504s slides were reviewed. The AMACR staining intensity was graded as negative, weak (focal apical cytoplasmic staining), or strong (diffuse strong cytoplasmic staining). CK 5/6 staining was retained when cytoplasmic staining was present. The percentage of cells stained by AMACR was estimated in high-grade prostatic intraepithelial neoplasia and in prostatic cancer glands; p63 staining was retained when nuclear staining was present. The percent of basal cells stained by CK 5/6 and p63 staining was estimated in normal prostate glands and ambiguous glands (Table 3 ).

\section{Statistical Analysis}

A statistical program (EPI INFO) was used to compare the sensitivity and specificity of the two antibodies (CK $5 / 6$ vs p63/p504s), and a $P$-value of $<0.05$ was considered statistically significant.

\section{Results}

\section{Histological Diagnosis}

All hematoxylin and eosin saffron slides were reviewed in this study. The initial diagnosis on usual staining was ambiguous lesions 'atypical small acinar proliferation, suspected to be but not diagnosed as malignant' in 104 cases, or prostate cancer in 101 cases, with small foci less than $1 \mathrm{~mm}$ in 14 cases. For the remainder, 19 cases consisted of high-grade prostatic intraepithelial neoplasia, two others were atypical adenomatous hyperplasias and 
34 cases showed benign features including clear-cell cribriform hyperplasia $(n=1)$, atrophic foci $(n=25)$, basal cell hyperplasia $(n=1)$ and transitional normal glands $(n=2)$.

The final diagnosis was based on combined use of the different antibodies, one (p504s) as a positive marker and the other (p63, CK 5/6) as a negative marker. In the case of CK $5 / 6$ or p63-/p504s + staining, the diagnosis of prostate cancer was retained; in the case of CK $5 / 6$ or $\mathrm{p} 63+/ \mathrm{p} 504 \mathrm{~s}-$ staining, the diagnosis of a benign lesion was proposed; in the case of CK 5/6 or p63+/p504s + staining, a diagnosis of high-grade prostatic intraepithelial neoplasia or atypical adenomatous hyperplasia was retained; and in the case of CK $5 / 6$ or p63-/p504s - staining the diagnosis of atypical small acinar proliferation suspected to be but not diagnosed as malignant was retained.

\section{CK 5/6}

The immunohistochemistry of CK $5 / 6$ was performed in 260 cases, and in one case prostate tissue

Table 3 Diagnosis before and after immunohistochemistry study

\begin{tabular}{lrrr}
\hline & Initial & CK $5 / 6$ & $p 63 / p 504 s$ \\
\hline ASAP & 104 & 59 & 11 \\
Prostate cancer & 101 & 128 & 150 \\
Benign lesions & 34 & 43 & 60 \\
AAH & 2 & 2 & 5 \\
HG PIN & 19 & 28 & 34 \\
\hline
\end{tabular}

ASAP, atypical small acinar proliferation; AAH, atypical adenomatous hyperplasia; HG PIN, high-grade prostatic intraepithelial neoplasia. was not available. Basal cell staining was observed in $86 \%$ of the tested cases $(222 / 259)$, with weaker expression in formaldehyde fixative solutions compared to Bouin or AFA $(P<0.0001)$ (Figure 1). No basal cells were observed in prostatic carcinomas (Figure 2). In high-grade prostatic intraepithelial neoplasias, the persistence of basal cells was observed in $20-30 \%$ of glands. In atrophic or atypical adenomatous hyperplasias persistent basal cells were present (Figure 4). In the 104 ambiguous lesions, CK 5/6 immunostaining in conjunction with histological standard histology led us to propose a definite benign diagnosis in the nine cases with persistence of basal cells. In nine cases, a diagnosis of high-grade prostatic intraepithelial neoplasia or atypical adenomatous hyperplasia was proposed; in 27 cases a diagnosis of small focus prostatic carcinoma was proposed; and in the 59 remaining cases, the diagnosis of persistent atypical small acinar proliferation and suspected but undiagnosed malignancy was retained.

\section{p504s/p63}

Immunohistochemistry of p504s/p63 was performed in 260 cases, and in one case no prostate tissue was available for immunostaining. In normal glands, p63 reacted with basal cells in $98 \%(254 / 259)$ of cases, with weak $(9 \%)$, moderate $(11 \%)$ or strong $(78 \%)$ nuclear staining (Figure 1). The intensity of nuclear staining was independent of the fixative technique $(P=0.1)$. In atrophic and benign lesions, $10-100 \%$ persistent basal cell staining was observed with p63 (Figure 4). All prostatic carcinomas were negative for p63.
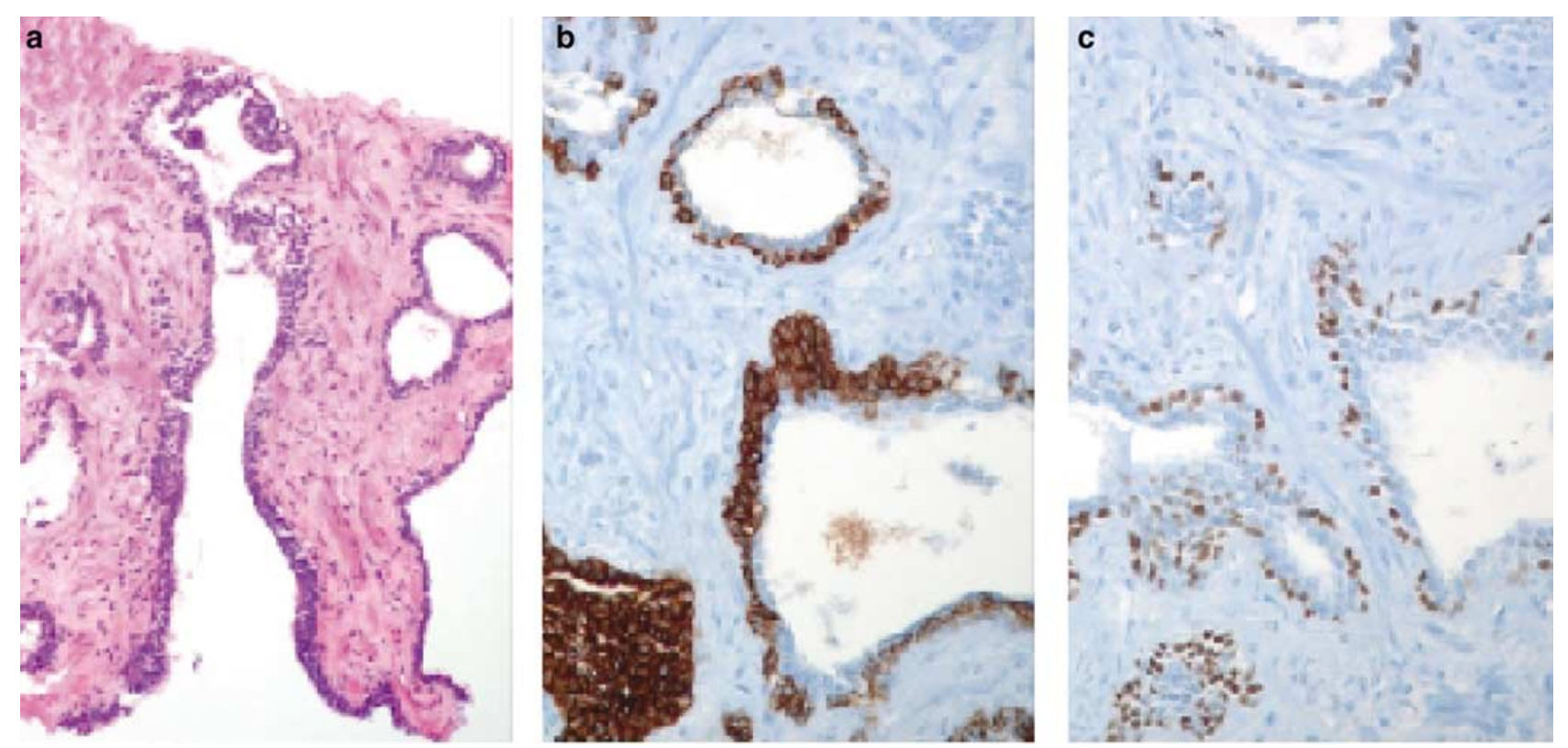

Figure 1 Normal prostate glands with basal cell markers: cytoplasmic staining with CK 5/6 (b) and nuclear staining with p63 (c). (a) HES, (b) CK 5/6, (c) p63/p504s. 

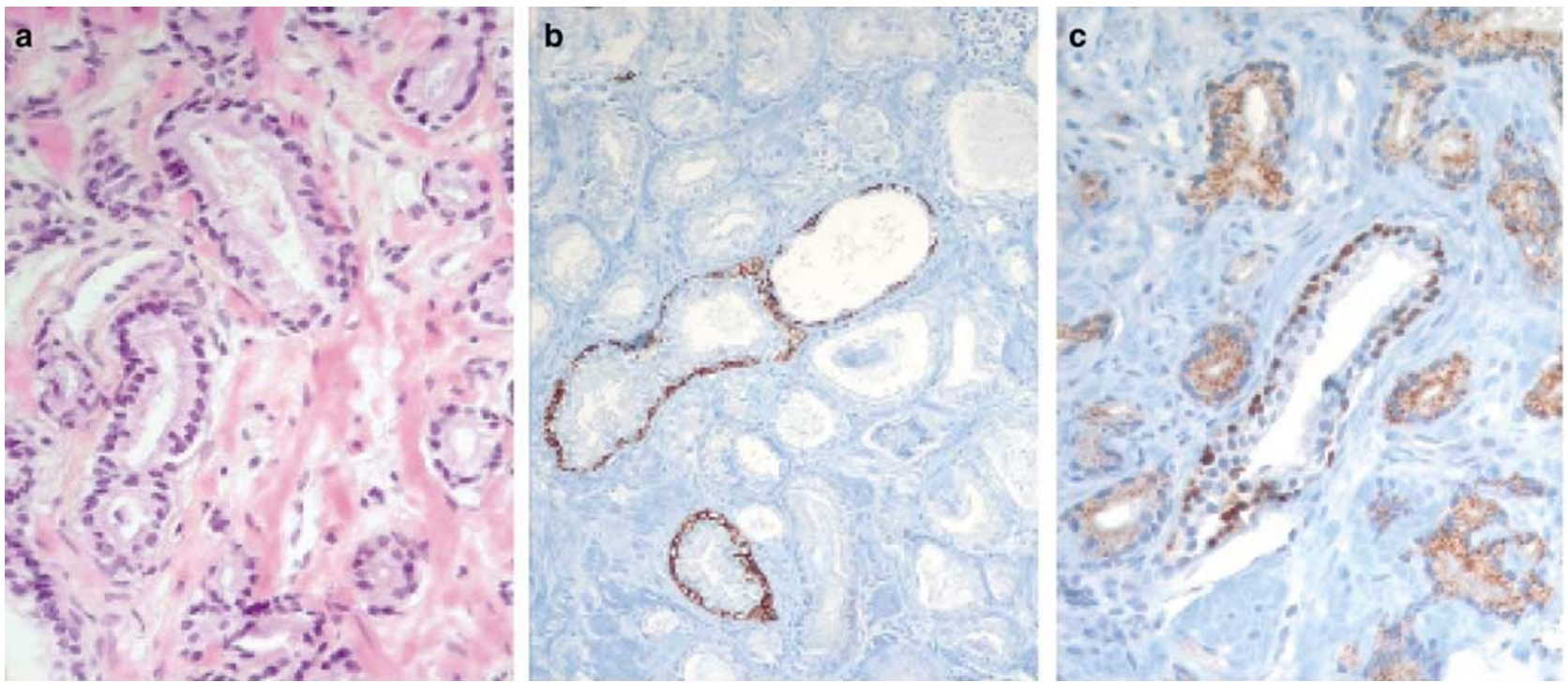

Figure 2 Prostatic cancer: persistent basal cells staining with CK 5/6 (b) and p63 (c). Cytoplasmic staining with p504s (c). (a) HES, (b) CK 5/6, (c) p63/p504s.

p504s reacted with only $2 \%$ of normal glands (4/ 260) (Figure 9) with a focal weak staining and $97 \%$ of prostatic cancer showed AMACR overexpression (Figure 2) with a heterogeneous staining pattern from weak $(30 \%)$ or moderate $(31 \%)$ to strong (36\%) intensity, independently of the Gleason score $(P=0.29)$, and the fixative technique $(P=0.27)$. In cases of atypical adenomatous hyperplasia or highgrade prostatic intraepithelial neoplasia, intracytoplasmic p504s staining was present in $70 \%$ of prostatic cells, associated with persistence of the basal cell layer with p63 (Figure 3).

Use of the p504s/p63 cocktail enabled a firm diagnosis in 89\% of ambiguous lesions: 47\% (49/ 104) were $\mathrm{p} 63-/ \mathrm{p} 504 \mathrm{~s}+$ and were diagnosed as minimal focus cancers (Figures 5 and 7); 25\% (26/ 104) were $\mathrm{p} 63+/ \mathrm{p} 504 \mathrm{~s}-$ and were diagnosed as benign lesions; and $17 \%(18 / 104)$ were p63+/ p504s + and were diagnosed as high-grade prostatic intraepithelial neoplasias in 14\% (15/104) or atypical adenomatous hyperplasias in 3\% (3/104) (Table 4). The remaining $11 \%(11 / 104)$ were p63-/p504scases and were retained as persistent atypical small acinar proliferations with a suspected but unconfirmed diagnosis of malignancy (Figure 6).

\section{Sensitivity and Specificity}

After immunohistochemical staining (Table 3), the final diagnosis was prostatic carcinoma in 151 cases, high-grade prostatic intraepithelial neoplasia in 30 cases, atypical small acinar proliferation with suspected but unconfirmed diagnosis of malignancy in 14 cases, and benign lesion in 62 cases, with atrophy in 42 cases and atypical adenomatous hyperplasia in three cases. When we compared the various antibodies in order to differentiate benign from malignant lesions, we observed a superiority in terms of sensitivity for p63/p504s (98\%) and p63 (87\%) over CK 5/6 (57\%), and specificity for p63/ p504s $(95 \%)$ and p63 (81\%) over CK 5/6 (88\%) (Table 5). Concerning ambiguous lesions, with CK $5 / 6$ only $53 \%(55 / 104)$ were correctly classified, while with the p63/p504s cocktail, 89\% (93/104) were correctly diagnosed.

\section{Discussion}

Histological confirmation of prostate cancer sometimes remains difficult to assert. The difficulty with needle biopsy arises when only a few malignant glands (small focus carcinomas, minute carcinomas) or histological benign mimics of cancer are present (Table 3). In light of these histologic features, an initial diagnosis based on standard histological staining of atypical small acinar proliferation suspected of malignancy but not diagnosed can be made. The diagnosis of such indeterminate cases concerns $1.5-9 \%$ of prostatic biopsies. ${ }^{9}$ One feature distinguishing benign cancer mimics from cancer and prostate cancer is that benign glands contain basal cells that are absent in cancerous glands, and pathologists often use immunohistochemical markers to label basal cells. ${ }^{2}$ In the presence of an ambiguous lesion, pathologists could use immunohistochemistry retrieval with anti-CK high-molecularweight antibody CK 903 to prove the absence of basal cells. ${ }^{2,10}$ In our study, the 3.5\% (59/1640) rate of ambiguous lesions after basal cell marker staining (CK $5 / 6$ ) is quite similar to the $1.1-6 \%$ reported by Iczkowski et $a l^{9}$ at three research facilities over a 10-year period.

The most commonly used marker is the highmolecular-weight CK 903 (34 $\beta$ E12). In 1984, Gown 

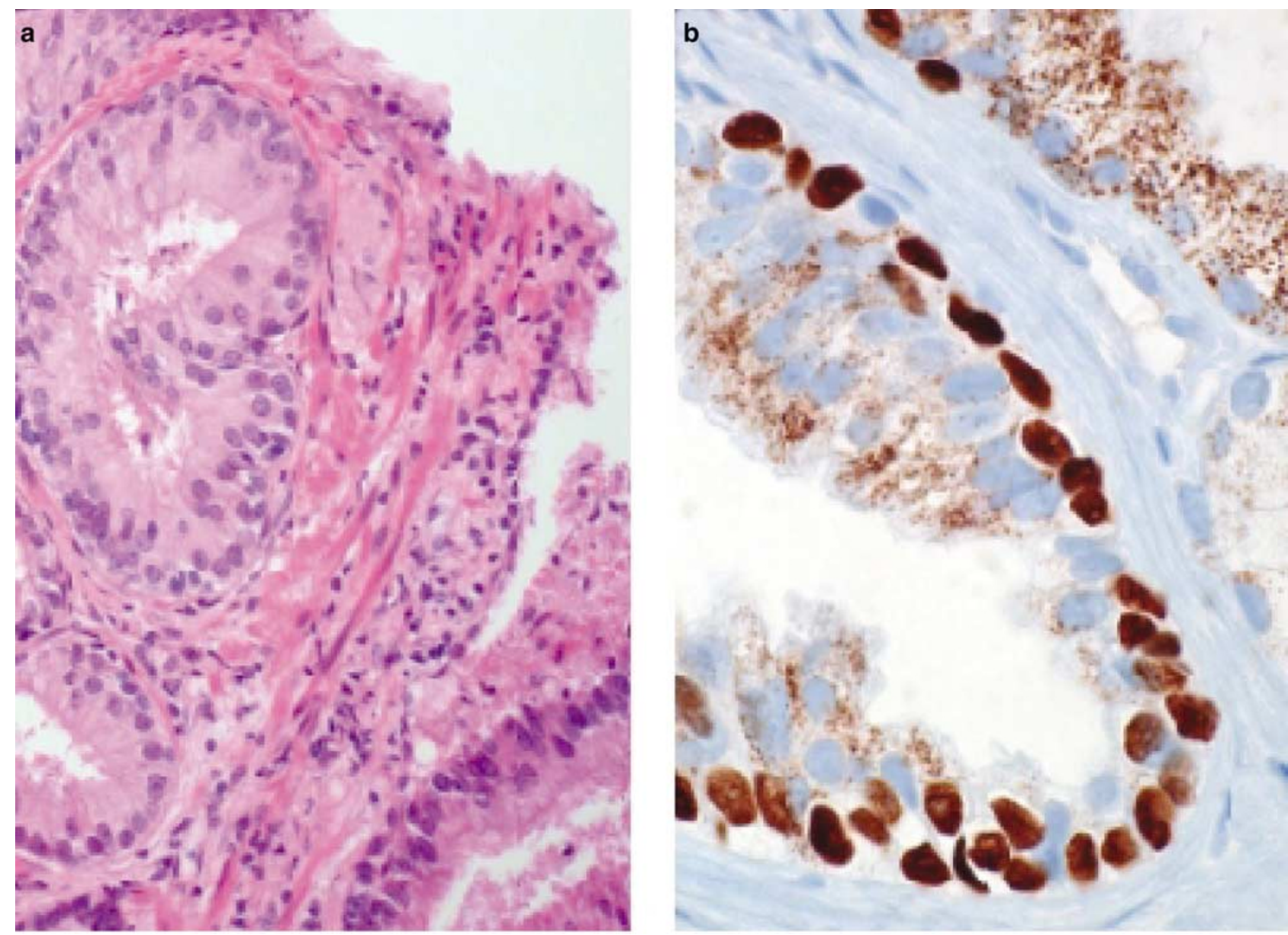

Figure 3 High-grade PIN: persistent basal cells staining with p63 and intracytoplasmic staining with p504s. (a) HES, (b) p63/p504s.
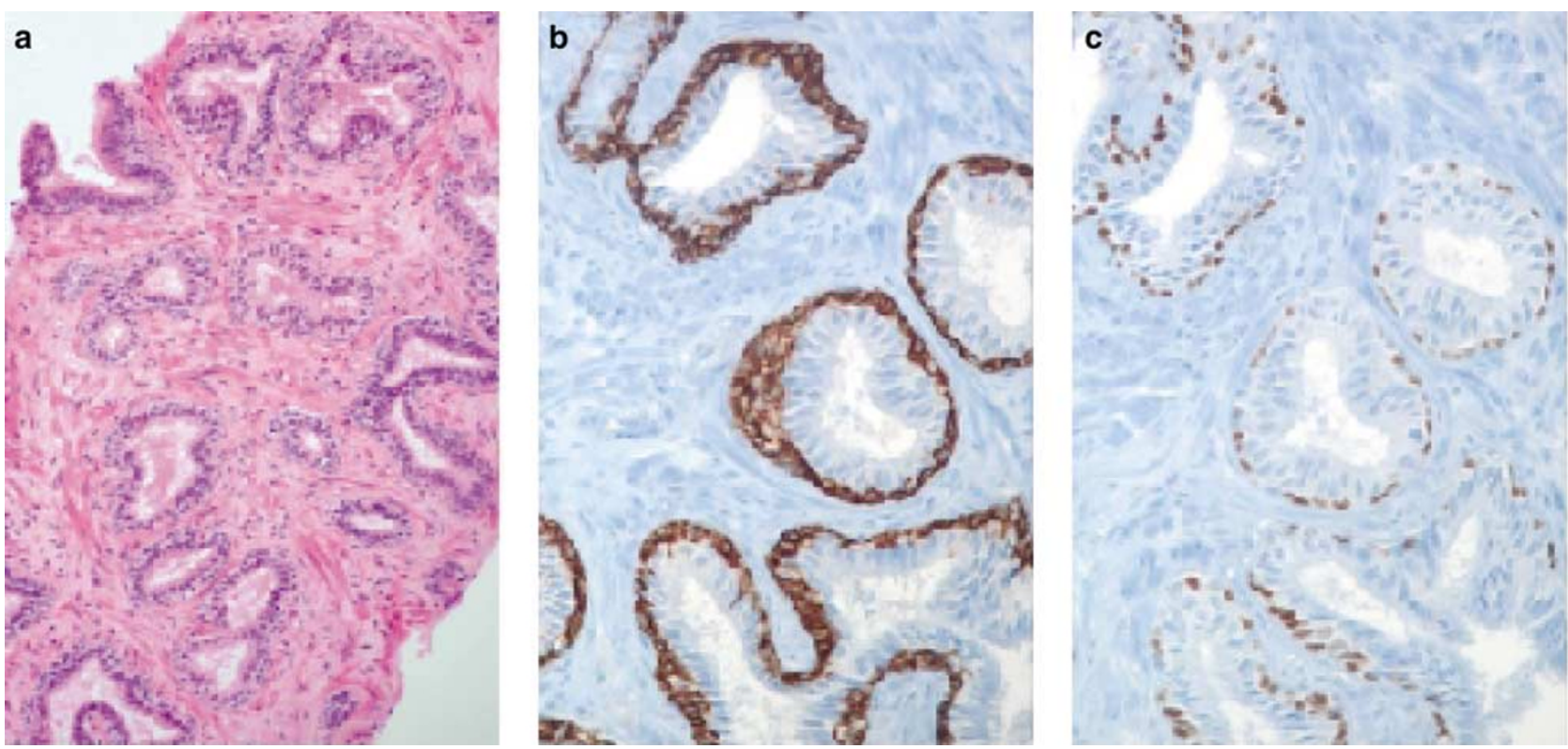

Figure 4 Basal cell hyperplasia: basal cell cytoplasmic staining with CK 5/6 (b) and nuclear staining with p63 (c). (a) HES, (b) CK 5/6, (c) $\mathrm{p} 63 / \mathrm{p} 504 \mathrm{~s}$. 
and Vogel $^{11}$ first reported results of $34 \beta \mathrm{E} 12$ as a marker of prostatic basal cells. Since then, numerous studies have shown that the systematic application of anti-CK 903 permitted diagnostic confirmation in $58 \%$ of the cases, restored a correct diagnosis in $18 \%$ of doubtful cases, and changed the diagnosis in $2 \%$ of the cases. ${ }^{10-12}$ However, application of this antibody is not practical in $8 \%$ of the cases. ${ }^{13}$ Indeed, we observed the disappearance of the layer of basal cells as follows: $11 \%$ in cases of atrophy, $12 \%$ in cases of basal cell hyperplasia and $10-90 \%$ in cases of atypical adenomatous hyperplasia. ${ }^{10}$ Despite the use of new methods applied to immunohistochemical techniques, including that of the Automate (Ventana, Dako), that is, automation of heat antigenic restoration, this antibody remains difficult to use, especially with Bouin's fixation, which requires technical subtleties for antigenic restoration, such as protease digestion, heat recovery, developer systems, and even, in some cases, all three. ${ }^{12-14}$

Recently, CK 5/6 has been reported to be a new marker of prostatic basal cells, with fewer unsatisfactory results and better sensitivity and specificity than CK 903. ${ }^{15}$ CK 5/6, normally expressed by complex epithelium, is a marker of mesothelial cells, malignant mesothelioma, as well as pancreatic, bile tract and mammary carcinomas. ${ }^{16,17}$ This antibody reacts with prostatic basal cells, but not with prostatic tumoral cells nor with high-grade

Table 4 Evaluation of ASAP with different antibodies $(n=104)$

\begin{tabular}{lcccc}
\hline & Prostate cancer & Benign lesion & AAH/PIN & ASAP \\
\hline CK 5/6 & $27(26 \%)$ & $9(8 \%)$ & $9(8 \%)$ & $59(57 \%)$ \\
p504s & $46(44 \%)$ & $29(28 \%)$ & $23(22 \%)$ & $6(6 \%)$
\end{tabular}

AAH, atypical adenomatous hyperplasia; PIN, prostatic intraepithelial neoplasia; ASAP, atypical small acinar proliferation. prostatic intraepithelial neoplasias, independent of the timing delay and the type of fixative (formalin $v s$ Bouin's solution). ${ }^{15}$ CK 5/6 does not require complex antigenic restoration and presents a sensitivity of $97-100 \%$ and a specificity of $62 \%$, which is better than the $40 \%$ observed with CK $903 .{ }^{15}$ In our experience, in the case of ambiguous lesions, CK 5/6 was more effective than CK 903.

The p63 antibody is a recently developed antibody to prostate basal cells. ${ }^{4}$ Protein p63, which shares homology with the suppressor gene of tumor p53, seems to play a critical role as a regulator of growth and development of cutaneous epithelium, uterine cervix, breast and the urogenital tract, and in particular, of prostate development. ${ }^{18}$ Signoretti et $a l^{3}$ first individualized the $\Delta \mathrm{Np} 63$ isoform in prostate basal cells and demonstrated the crucial role of p63 in development of the prostate. Subsequently, numerous authors showed that p63 was expressed by basal cells in normal prostatic glands. ${ }^{4,19,20}$ demonstrated that the vast majority (89-94\%) of prostate carcinomas do not express p63 ${ }^{4}$ and that p63 immunostaining represents a novel adjuvant method for facilitating pathologic diagnosis in prostate needle biopsy. Further studies indicate that p63 is not expressed by prostate tumor cells and can therefore be used in the same way as CK 903 in the diagnosis of atypical small acinar proliferation suspected to be, but not diagnosed as, malignant. ${ }^{2}$ Our results concerning the total absence of basal cells in carcinomatous glands are comparable to those reported by Zhou et al, ${ }^{21}$ which showed that p63 was not expressed in prostate cancer.

Our results reveal high expression of p63 in 100\% of normal basal cells and confirm the superiority on CK 5/6, with an improvement in specificity (87 vs $57 \%$ ) along with a slight decrease in sensitivity (81 vs $88 \%$ ). This confirms results of Shah et al, ${ }^{2}$ who underlined that p63 was more specific than CK 903, as did Zhou et $a l,{ }^{22}$ who showed the advantage of
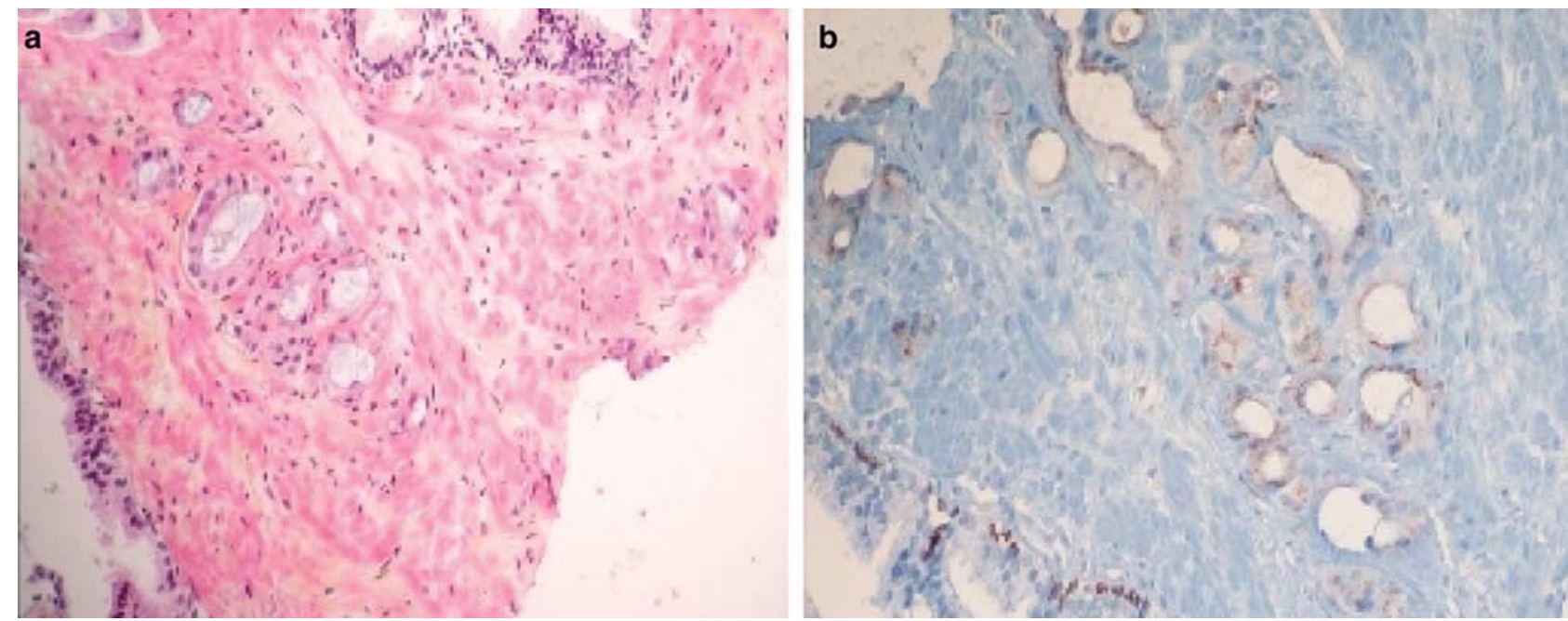

Figure 5 Ambiguous lesion: the normal glands are p63+/p504s-; and the atypical glands are p63-/p504s +. Final diagnostic: small focus of prostatic carcinoma. (a) HES, (b) p63/p504s. 

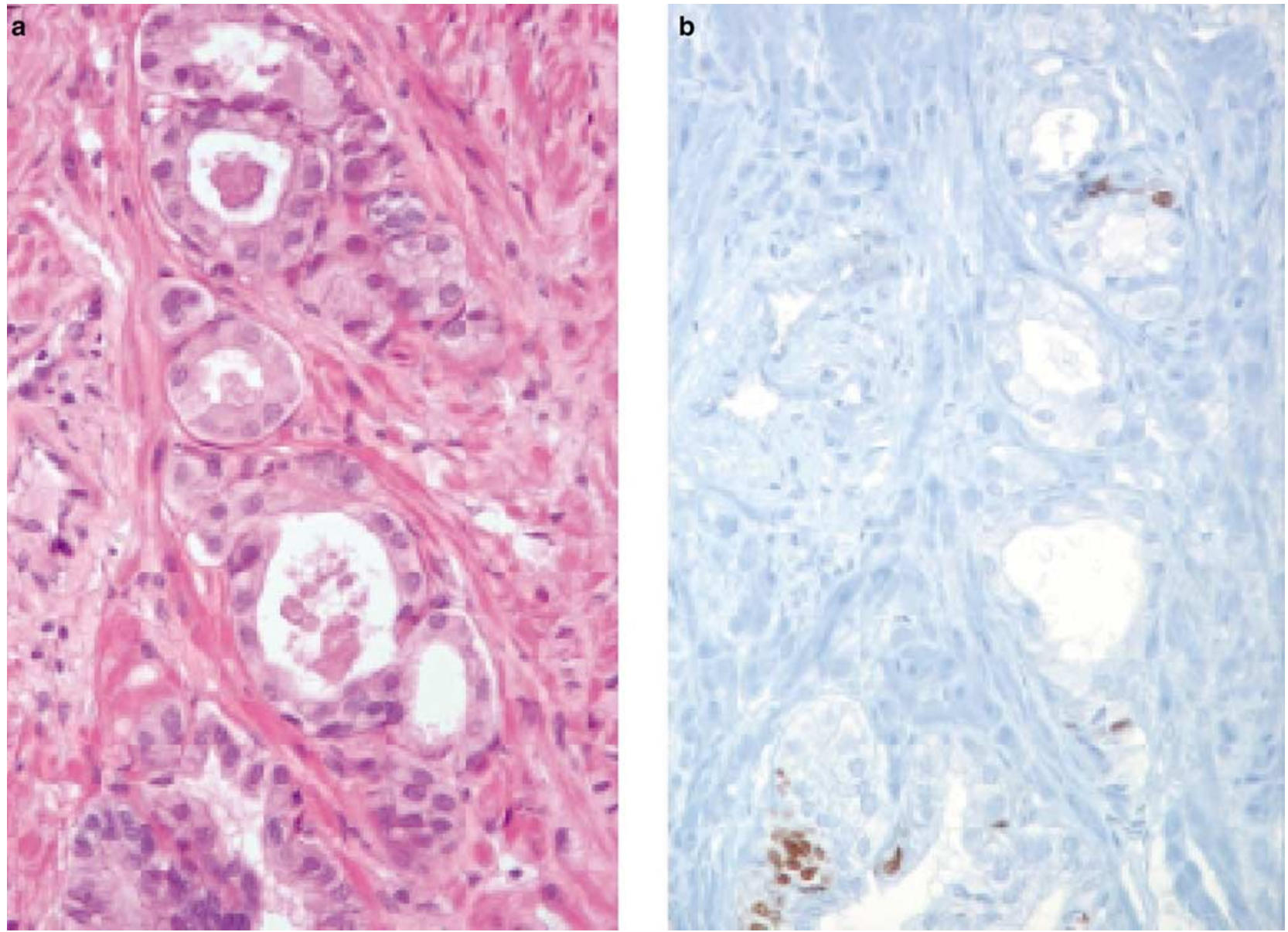

Figure 6 Ambiguous lesion: the normal glands are p63+/p504s-; and the atypical glands are p63-/p504s-. Final diagnostic: ASAP. (a) HES, (b) p63/p504s.

conjugating the two antibodies (34 $\beta \mathrm{E} 12$ and $\mathrm{p} 63)$ for the detection of basal cells. The persistence of basal cells observed in $70 \%$ of atypical adenomatous hyperplastic glands confirms results reported by Shah et $a l,{ }^{2}$ who observed the obstinacy of moderate nuclear marking to intense nuclear staining of p63 in $70 \%$ of basal cells.

More recently, AMACR, a cytoplasmic protein recently identified by cDNA library subtraction in conjunction with high-output microarray analysis of prostatic carcinoma, ${ }^{6,23}$ has been considered as a marker that is substantially upregulated in prostate cancer. AMACR overexpression has been detected in many human tumors such as colon and mammary tumors, malignant melanomas and papillary renal carcinomas. ${ }^{24-26}$ AMACR is known to be involved in the beta-oxidation of branched-chain fatty acids and fatty acid derivatives. ${ }^{27,28}$ AMACR has been located in peroxisomes and mitochondria and in human cells, and expression of the enzyme is strongly expressed in normal liver and kidney cells. ${ }^{29}$ The racemic mixtures of branched fatty acids and fatty acid derivatives must first be converted by AMACR into peroxisomes, and mitochondria into $(S)$ isomers, before undergoing beta-oxidation. ${ }^{28}$ Mitochon- drial beta-oxidation of fatty acids donates electrons to the respiratory chain coupled with phosphorylation of ADP to ATP. Peroxisomes transform branched-chain fatty acids by acyl-CoA oxidase with the production of oxidized substrate and hydroxide hydrogen. ${ }^{27,28}$

Western blot analysis demonstrated 36-fold overexpression of p504s in prostatic carcinomas when compared with benign glandular tissues. ${ }^{6}$ Using a highly specific antibody (p504s) directed against the enzyme, Jiang et $a l^{30}$ found that strong immunostaining for the enzyme was consistently present in prostatic carcinoma and in high-grade prostatic intraepithelial neoplasia of the peripheral zone of the prostate. In contrast, immunostaining was not detected in $88 \%$ of the 173 cases of benign hypertrophic hyperplasia, whereas in the remaining $12 \%$ only focal weak immunostaining was present. ${ }^{30}$ Therefore, the investigators concluded that p504s was a highly specific and sensitive marker of prostatic carcinoma. Over the past 2 years, many studies have reported extensive upregulation of AMACR at the protein and transcript levels in high-grade prostatic intraepithelial neoplasia and prostate cancer. ${ }^{5,8,10}$ 

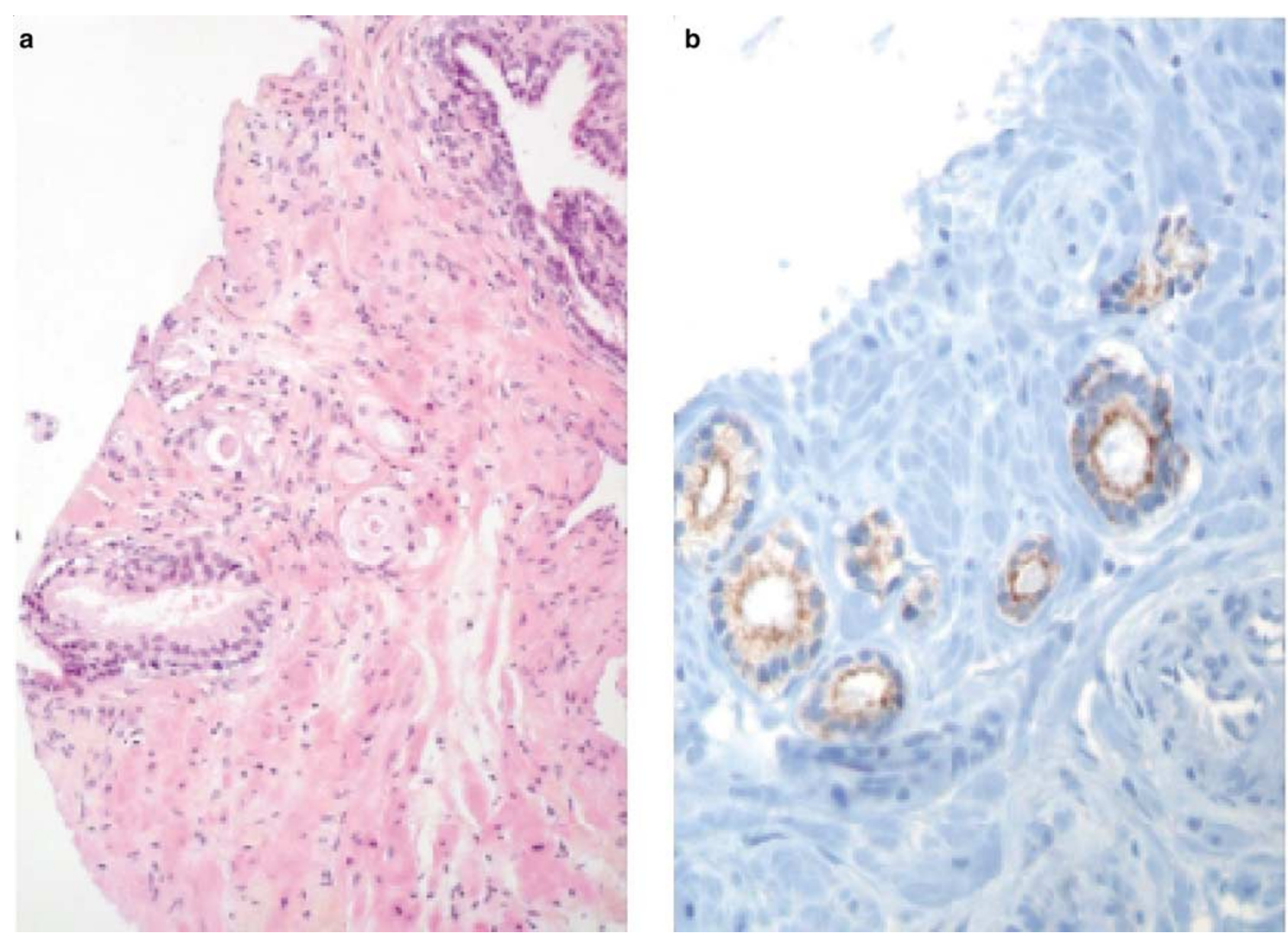

Figure 7 ASAP: the atypical glands are p63-/p504s +. Final diagnostic: small focus of prostatic carcinoma. (a) HES, (b) p63/p504s.

Table 5 Sensitivity and specificity of different antibodies

\begin{tabular}{lccc}
\hline & $C K 5 / 6-(\%)$ & $p 63-(\%)$ & $p 63+/ p 504-(\%)$ \\
\hline Sensitivity & 56.9 & 86.3 & 97.6 \\
Specificity & 87.8 & 81.4 & 95.2 \\
NPV & 60.4 & 60.3 & 96.8 \\
PPV & 86.2 & 94.8 & 96.3 \\
\hline
\end{tabular}

NPV, negative predictive value; PPV, positive predictive value.

As previously reported in our series, we found that the positivity of p504s was independent of the Gleason score or fixative mode. Beach et $a l^{5}$ found $82 \%$ p504s expression in prostate cancer whatever the morphological aspect, that is, classical, intraductal, mucinous, pseudohyperplastic or atrophic, degree or differentiation, Gleason score or additional treatment such as hormone therapy or radiotherapy. But if we considered p504s intensity of immunostaining, we found only $67 \%$ moderate and intense cytoplasmic staining and weak staining in $31 \%$ of our cases. This could explain our better score of immunostaining compared to the $80-85 \%$ p504s expression in prostate cancer usually reported.
In high-grade prostatic intraepithelial neoplasia, prostatic cells expressed p504s in 20-80 \% of cases, with a granular intracytoplasmic pattern. This confirms the results of Beach et al, ${ }^{5}$ who reported expression in $32 \%$ of the cases. ${ }^{5}$ In our experience, we observed a weaker intensity of expression of p504s in high-grade prostatic intraepithelial neoplasias compared to prostatic carcinomas.

In only four cases $(2 \%)$ did we observe granular intracytoplasmic expression by rare normal prostatic cells (Figure 9). The application, in our series, of coexistent antibody again basal cells (p63) demonstrated that these glands had fewer basalstained than nonpositive p504s normal glands and led us to believe that this could be interpreted as low-grade prostatic intraepithelial neoplasia (Figure 8). This result is not concordant with the $21 \%$ reported by Beach et al..$^{5}$ Like those authors, we also observed that this expression is generally focal and is weaker than the positivity of the tumoral prostatic glands. ${ }^{5}$ The increased AMACR expression by some normal prostatic glands could reflect upregulation of metabolic pathways of prostate glands during the carcinogenic process. ${ }^{8}$ This finding is quite similar to that of Leav et $a{ }^{31}{ }^{31}$ who found that overexpression of AMACR was present in transitional lesions 

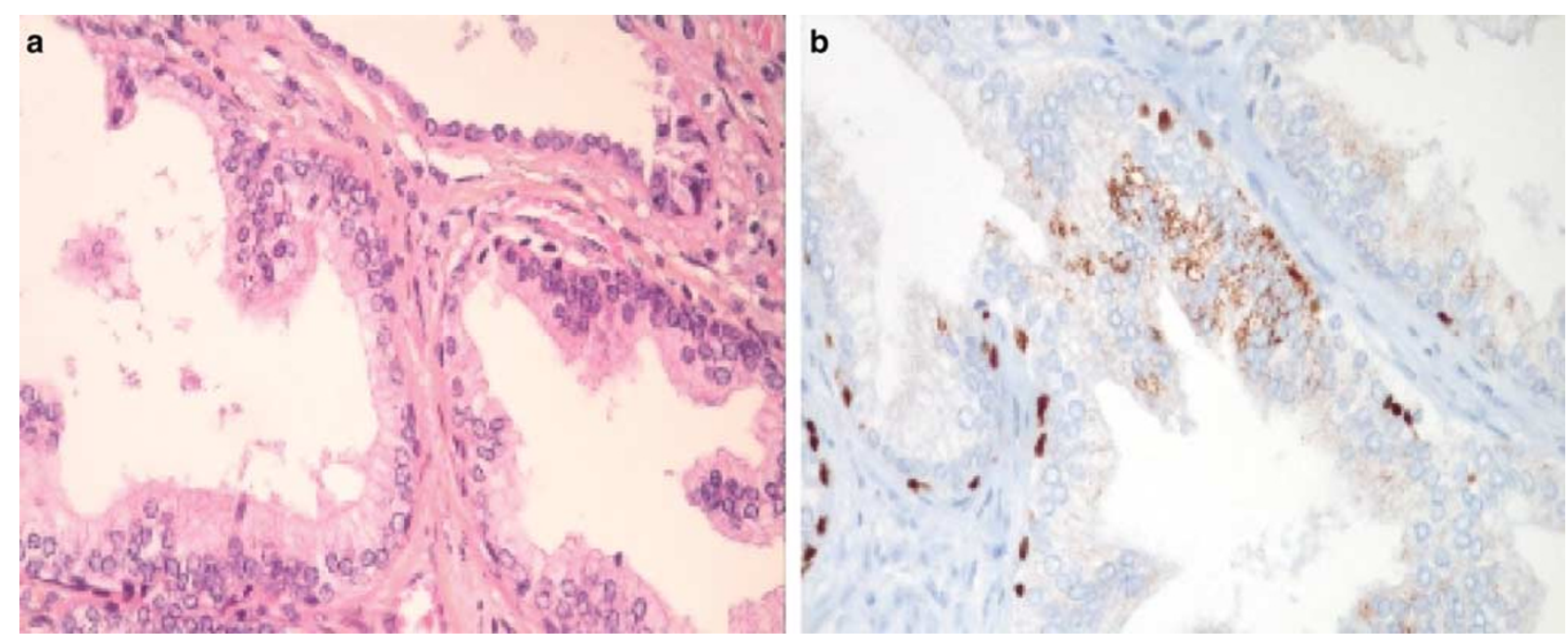

Figure 8 Low-grade PIN: the glands are p63+/p504s +. (a) HES, (b) p63/p504s.
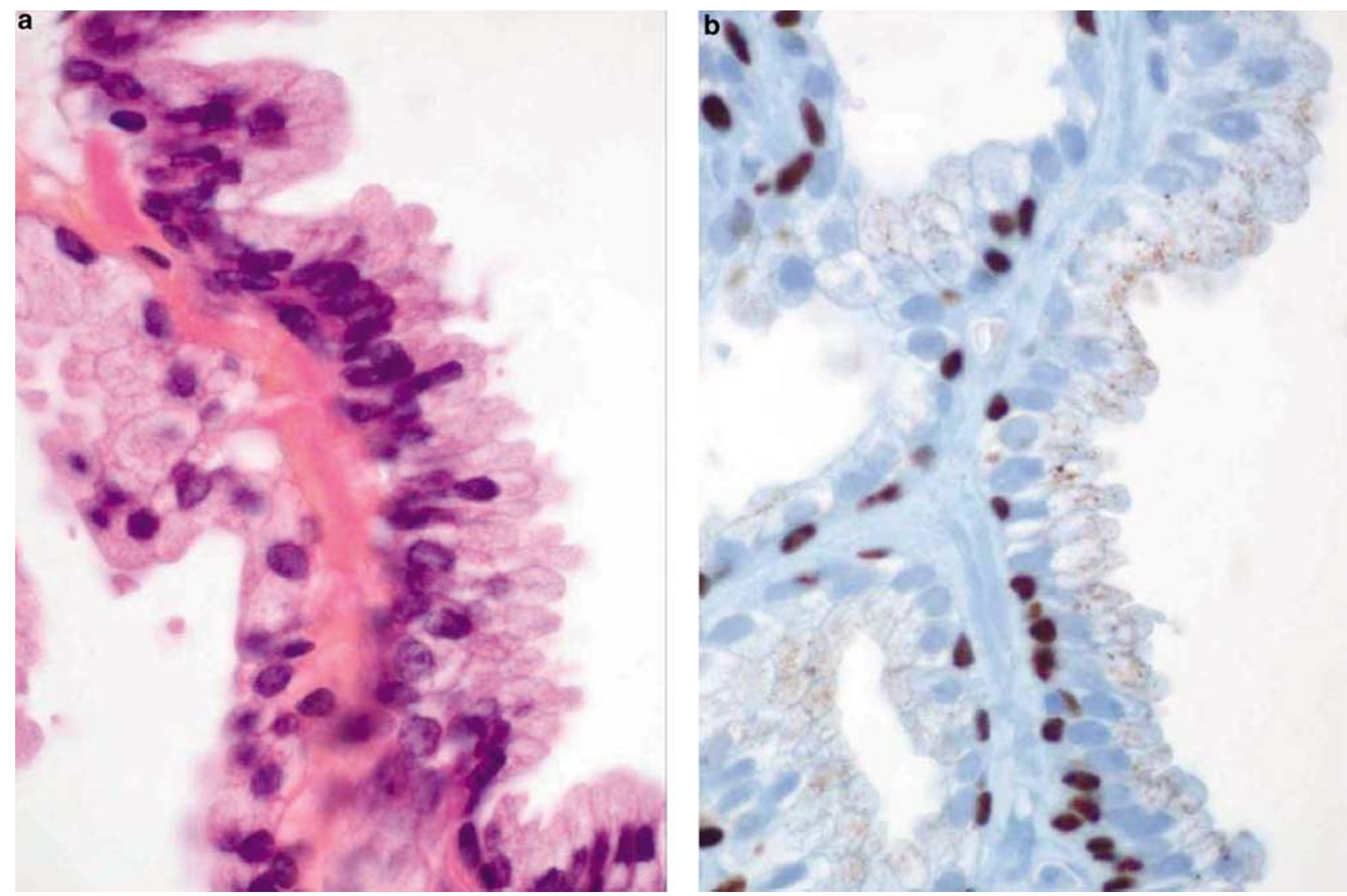

Figure 9 Normal glands with weak apical intracytoplasmic positivity with p504s and disruption of p63 basal cell layer: this could represent the primitive upregulation of metabolic pathways of prostate glands during carcinogenic process. (a) HES, (b) p63/p504s.

in benign hypertrophic hyperplasia nodules along the transitional zone carcinomas, and confirmed that p504s is a useful marker of neoplastic transformation in the prostate gland. Increased AMACR expression very likely reflects the upregulation of metabolic pathways of normal glands. ${ }^{31}$ These results are also in agreement with the study of Jiang et $a l,{ }^{30}$ who found numerous benign hypertrophic hyperplastic nodules negative for p504s staining, indicating that cancer development is restricted to a subset of hypertrophic lesions, which may be identified by the enhanced expression of AMACR. 
Our results confirm the absence of expression of p504s in cases of atrophy, postatrophic hyperplasia, transitional metaplasia and basal cell hyperplasia. ${ }^{30}$ The high expression observed in more than $70 \%$ of the prostate cells in atypical adenomatous hyperplasia confirms the results reported by Yang et al. ${ }^{32}$

With p504s, our results in terms of sensitivity and specificity (98 and 95\%) are comparable to those reported in the literature. In our experience, the high specificity and sensitivity of p504s are better than those observed with p63, CK 903 or CK 5/6 (Table 5) to detect prostate cancer in foci of ambiguous lesions such as atypical small acinar proliferations suspected of malignancy. The association of p504s with p63 as a useful marker of neoplastic transformation in the prostate gland has been proposed by several groups as a potential marker of prostatic tumoral cells in a diagnostic context, with increased sensitivity of up to $97 \%$ and specificity of up to $100 \%$, both in tissue array and standard biopsies. $^{24,33,34}$ Like Shah et al, who underlined the interest of associating p63 immunostaining with CK $903,{ }^{17}$ and like Luo et $a l^{8}{ }^{8}$ we believe that, in a simple assay, the association of the two antibodies p504s and p63, one (p504s) as a positive marker and the other (p63) as a negative marker, greatly facilitates the identification of malignant prostate cells, leading to a decrease in the risk of false negatives, an increase in diagnostic precision, and improved sensitivity and specificity (Table 5). In our experience, $89.4 \%$ of the atypical small acinar proliferation group can be diagnosed using a cocktail of the two antibodies. In our study, this led to a final diagnosis of benign lesions in $25 \%$ of cases, minimal cancer focus in $47.11 \%$, high-grade prostatic intraepithelial neoplasia in $14.4 \%$ and atypical adenomatous hyperplasia in $2.8 \%$ of our cases. Furthermore, the automatic processor Ventana Nexes ${ }^{\circledR}$ enabled us to carry out a successful immunostaining procedure with two-step primary antibody incubation and a secondary mixed antirabbit and anti-mouse antibody, in the same run. This considerably reduced the time of processing and enabled this detection in a routine procedure. This technique of coupling the two antibodies at the same time decreased the number of slides needed, the technical time, and in case of ambiguity, it reduced the percentage of inconclusive interpretations and additional biopsies.

\section{Conclusion}

In the last 2 years, new antibodies against prostatic basal cells (CK 5/6, p63) and prostatic tumor cells (p504s) have proven to be advantageous. Our results show that, for ambiguous lesions such as small foci of prostatic carcinoma or atypical small acinar proliferation suspected to be malignant but not diagnosed, immunostaining with the p504s/p63 cocktail improved diagnostic performance, helped to avoid carrying out new biopsies, and seemed more relevant than the application of antibody against high-weight-molecular CK $(34 \beta \mathrm{E} 12$ or CK 5/6) alone. The cost of immunohistochemical techniques remains lower than a new series of biopsies.

\section{References}

1 Jani AB, Hellman S. Early prostate cancer: clinical decision-making. Lancet 2003;361:1045-1053.

2 Shah RB, Zhou M, LeBlanc M, et al. Comparison of the basal cell-specific markers, 34betaE12 and p63, in the diagnosis of prostate cancer. Am J Surg Pathol 2002;26:1161-1168.

3 Signoretti S, Waltregny D, Dilks J, et al. p63 is a prostate basal cell marker and is required for prostate development. Am J Pathol 2000;157:1769-1775.

4 Parsons JK, Gage WR, Nelson WG, et al. p63 protein expression is rare in prostate adenocarcinoma: implications for cancer diagnosis and carcinogenesis. Urology 2001;58:619-624.

5 Beach R, Gown AM, De Peralta-Venturina MN, et al. p504s immunohistochemical detection in 405 prostatic specimens including 376 18-gauge needle biopsies. Am J Surg Pathol 2002;26:1588-1596.

$6 \mathrm{Xu} \mathrm{J}$, Stolk JA, Zhang X, et al. Identification of differentially expressed genes in human prostate cancer using subtraction and microarray. Cancer Res 2000;60:1677-1682.

7 Luo JH. Gene expression alterations in human prostate cancer. Drugs Today (Barc) 2002;38:713-719.

8 Luo J, Zha S, Gage WR, et al. Alpha-methylacyl-CoA racemase: a new molecular marker for prostate cancer. Cancer Res 2002;62:2220-2226.

9 Iczkowski KA, Chen HM, Yang XJ, et al. Prostate cancer diagnosed after initial biopsy with atypical small acinar proliferation suspicious for malignancy is similar to cancer found on initial biopsy. Urology 2002;60:851-854.

10 Helpap B, Kollermann J, Oehler U. Limiting the diagnosis of atypical small glandular proliferations in needle biopsies of the prostate by the use of immunohistochemistry. J Pathol 2001;193:350-353.

11 Gown AM, Vogel AM. Monoclonal antibodies to human intermediate filament proteins. II. Distribution of filament proteins in normal human tissues. Am J Pathol 1984;114:309-321.

12 Varma M, Linden MD, Amin MB. Effect of formalin fixation and epitope retrieval techniques on antibody 34betaE12 immunostaining of prostatic tissues. Mod Pathol 1999;12:472-478.

13 Hedrick L, Epstein JI. Use of keratin 903 as an adjunct in the diagnosis of prostate carcinoma. Am J Surg Pathol 1989;13:389-396.

14 Iczkowski KA, Cheng L, Crawford BG, et al. Steam heat with an EDTA buffer and protease digestion optimizes immunohistochemical expression of basal cell-specific antikeratin 34betaE12 to discriminate cancer in prostatic epithelium. Mod Pathol 1999;12:1-4.

15 Abrahams NA, Ormsby AH, Brainard J. Validation of cytokeratin $5 / 6$ as an effective substitute for keratin 903 in the differentiation of benign from malignant glands in prostate needle biopsies. Histopathology 2002;41:35-41. 
16 Carella R, Deleonardi G, D’Errico A, et al. Immunohistochemical panels for differentiating epithelial malignant mesothelioma from lung adenocarcinoma: a study with logistic regression analysis. Am J Surg Pathol 2001;25:43-50.

17 Chu PG, Weiss LM. Keratin expression in human tissues and neoplasms. Histopathology 2002;40: 403-439.

18 Yang A, McKeon F. p63 and p73: p53 mimics, menaces and more. Nat Rev Mol Cell Biol 2000;1:199-207.

19 Davis LD, Zhang W, Merseburger A, et al. p63 expression profile in normal and malignant prostate epithelial cells. Anticancer Res 2002;22:3819-3825.

20 Weinstein MH, Signoretti S, Loda M. Diagnostic utility of immunohistochemical staining for p63, a sensitive marker of prostatic basal cells. Mod Pathol 2002;15: 1302-1308.

21 Zhou M, Jiang Z, Epstein JI. Expression and diagnostic utility of alpha-methylacyl-CoA-racemase (p504s) in foamy gland and pseudohyperplastic prostate cancer. Am J Surg Pathol 2003;27:772-778.

22 Zhou M, Chinnaiyan AM, Kleer CG, et al. alphaMethylacyl-CoA racemase: a novel tumor marker over-expressed in several human cancers and their precursor lesions. Am J Surg Pathol 2002;26: 926-931.

23 Jiang Z, Woda BA, Rock KL, et al. p504s: a new molecular marker for the detection of prostate carcinoma. Am J Surg Pathol 2001;25:1397-1404.

24 Jiang Z, Fanger GR, Woda BA, et al. Expression of alpha-methylacyl-CoA racemase (p504s) in various malignant neoplasms and normal tissues: astudy of 761 cases. Hum Pathol 2003;34:792-796.

25 Jiang Z, Iczkowski KA, Woda BA, et al. p504s immunostaining boosts diagnostic resolution of 'suspicious' foci in prostatic needle biopsy specimens. Am J Clin Pathol 2004;121:99-107.

26 Tretiakova MS, Sahoo S, Takahashi M, et al. Expression of alpha-methylacyl-CoA racemase in papillary renal cell carcinoma. Am J Surg Pathol 2004;28:69-76.

27 Amery L, Fransen M, De Nys K, et al. Mitochondrial and peroxisomal targeting of 2-methylacyl-CoA racemase in humans. J Lipid Res 2000;41:1752-1759.

28 Evans AJ. alpha-Methylacyl CoA racemase (p504s): overview and potential uses in diagnostic pathology as applied to prostate needle biopsies. J Clin Pathol 2003;56:892-897.

29 Schmitz W, Albers C, Fingerhut R, et al. Purification and characterization of an alpha-methylacyl-CoA racemase from human liver. Eur J Biochem 1995;231: 815-822.

30 Jiang Z, Wu CL, Woda BA, et al. p504s/alphamethylacyl-CoA racemase: a useful marker for diagnosis of small foci of prostatic carcinoma on needle biopsy. Am J Surg Pathol 2002;26:1169-1174.

31 Leav I, McNeal JE, Ho SM, et al. alpha-Methylacyl-CoA racemase (p504s) expression in evolving carcinomas within benign prostatic hyperplasia and in cancers of the transition zone. Hum Pathol 2003;34:228-233.

32 Yang XJ, Wu CL, Woda BA, et al. Expression of alphamethylacyl-CoA racemase (p504s) in atypical adenomatous hyperplasia of the prostate. Am J Surg Pathol 2002;26:921-925.

33 Rubin MA, Zhou M, Dhanasekaran SM, et al. alphaMethylacyl coenzyme A racemase as a tissue biomarker for prostate cancer. JAMA 2002;287:1662-1670.

34 Luo J, Dunn TA, Ewing CM, et al. Decreased gene expression of steroid 5 alpha-reductase 2 in human prostate cancer: implications for finasteride therapy of prostate carcinoma. Prostate 2003;57:134-139. 